\title{
Exact magnetization plateaus and phase transitions in spin- $S$ Heisenberg antiferromagnets in arbitrary dimensions
}

\author{
V. Ravi Chandra and Naveen Surendran \\ Centre for High Energy Physics, Indian Institute of Science, Bangalore - 560 012, India
}

\begin{abstract}
We generalize a class of Heisenberg antiferromagnets in one, two and three dimensions, which have been shown to exhibit magnetization plateaus for spin- $\frac{1}{2}$. In a certain parameter range of the general model, which is formally defined in $D$ dimensions, we obtain the exact ground state(s) in the presence of an external magnetic field for arbitrary values of spin. In this range, the magnetization remains a constant as a function of the external field, except at some special values of the field where there is a jump from one plateau to the next. The plateaus are formed at certain specific fractions of the full magnetization which are determined by the spin and the lattice. Our general spin- $S$ result reproduces the known cases for spin- $\frac{1}{2}$ in various lattices. Furthermore, we argue that outside the exact regime, the mechanism for the plateau formation is different. This results in first order phase transitions along some of the plateaus as the coupling constant is varied. We rigorously show the existence of such transitions for some particular cases. Finally, we numerically analyze a spin-1 model in one dimension using exact diagonalization to obtain its complete phase diagram. It agrees with our analytic results.
\end{abstract}

PACS numbers: 75.10.Jm, 75.10.Pq, 75.30.Kz, 75.45.+j

\section{INTRODUCTION}

Many low-dimensional spin systems, especially in one dimension, respond discontinuously to the variations of an external magnetic field ${ }^{\underline{1}}$. In such systems, magnetization curve forms plateaus as a function of the field. In most cases, plateaus are continuously connected to one another, whereas a more interesting scenario is when there are abrupt jumps between two plateaus. This usually happens at high fields in systems with localized magnon excitations 2 . There is a class of models for which magnetization plateaus of the second type are formed via a simple mechanism. The basic feature of these models is that they consist of units of spins coupled together such that the total spin of each unit is conserved ${ }^{3.4 .5 .6 .7}$. The first of its kind was introduced and studied at zero field by Gelfand ${ }^{3}$. The system consists of coupled dimers with the following Hamiltonian (see Fig. \),

$$
H_{l}=J^{\prime} \sum_{i} \mathbf{S}_{1, i} \cdot \mathbf{S}_{2, i}+J \sum_{i}\left(\mathbf{S}_{1, i}+\mathbf{S}_{2, i}\right) \cdot\left(\mathbf{S}_{1, i+1}+\mathbf{S}_{2, i+1}\right) .
$$

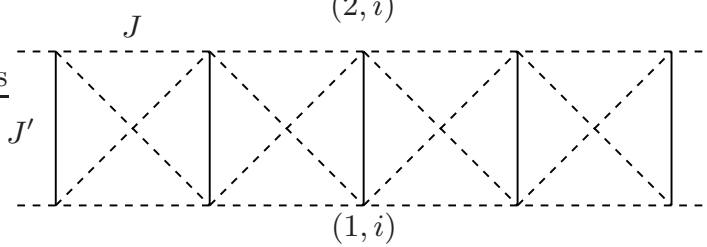

FIG. 1: The Gelfand ladder. The full lines represent the coupling $J^{\prime}$ and the dashed lines $J$.

Note that the total spin on each dimer $\left(S_{1, i}^{a}+S_{2, i}^{a}\right)$ is conserved. A whole lot of eigenstates can be trivially constructed for the above Hamiltonian. Any state in which each dimer has a specific total spin and is maximally polarized in the $z$-direction will be an eigenstate. Then, one expects the ground state to be a product of dimer-singlets for $J^{\prime}>>J$. A lower bound on $J^{\prime}$ such that the dimer-singlet is the ground state has been obtained by rewriting the Hamiltonian as sum of units consisting of three spins ${ }^{9}$, and is given by,

$$
J^{\prime} \geq \begin{cases}2 J, & \text { for } S=\frac{1}{2} \\ 2 J(S+1), & \text { for } S \geq 1\end{cases}
$$

For $S=\frac{1}{2}$, the ground state in fact remains to be the dimer-singlet for $J^{\prime}>1.4 J$. For $J^{\prime}<1.4 J$, all the dimers go into triplets and form the ground state of a spin-1 uniform chain. 
The lower-bound for $J^{\prime}$ given in (2) suggests that, the higher the spin, the stronger the dimer bond needs to be for the dimer-singlet to be the ground state. But it turns out that the dimer-singlet is quite robust even for very large spins. In this paper, we show that the lower bound on $J^{\prime}$ can be pushed down to $2 J$ for arbitrary values of spin, i.e., the bound given by (2) for $S=\frac{1}{2}$ is in fact true for all values of $S$.

Honecker, Mila and Troyer ${ }^{6}$ (from hereon referred to as HMT) have numerically studied the $S=\frac{1}{2}$ system in the presence of magnetic field. For $J^{\prime}>2 J$, the system starts with the dimer-singlet ground state at zero field and the magnetization per site $(M)$ is zero. The system remains in the dimer-singlet phase till a transition field $B_{c_{1}}=J^{\prime}$, at which one set of alternate dimers form triplets polarized in the direction of the magnetic field $\left(M=\frac{1}{2}\right)$. For further increase in the field strength, this state remains to be the ground state till a second transition field $B_{c_{2}}=2 J+J^{\prime}$, at which the rest of the dimers also become triplets and the system is fully polarized $(M=1)$. This behaviour has also been inferred on the basis of a strong coupling analysis $\stackrel{8}{*}$. For $J^{\prime}<2 J$, gapless phases come into play and the magnetization curve starts developing tails between plateaus before the latter disappear altogether. HMT also studied a three-leg ladder and obtained abrupt jumps between plateaus in some range of the parameter. Similar results for $S=\frac{1}{2}$ have been obtained for a modified Shastry-Sutherland model in two dimensions ${ }^{\underline{\underline{ }}}$ and a three-dimensional version of Gelfand ladder ${ }^{7}$.

In this paper, we define a general model in arbitrary dimensions which includes all the models mentioned above. In a certain regime of the parameter space, we obtain the exact ground state(s) in presence of magnetic field for arbitrary values of spin. The magnetization curve forms plateaus and they jump abruptly from one to another at certain special values of the field. The magnetization on the plateaus are some rational fractions of the full magnetization, which depend on the underlying spin and the lattice.

Outside the exact regime, we argue that the plateaus will survive in the immediate neighbourhood, quite possibly developing tails between successive ones. But the mechanism for plateau formation in this case is different from the exact regime and this results in first order phase transitions along some of the plateaus. We rigorously show the existence of such transitions for some special cases. Finally, we numerically analyse the ladder defined in (1D) for $S=1$ using exact diagonalization. We obtain the complete phase diagram which confirms our analytic results.

\section{THE GENERAL MODEL AND THE EXACT GROUND-STATES}

HMT have defined a general $n$-leg ladder in one dimension. We generalize further and formally define Hamiltonians on an arbitrary bipartite lattice in $D$ dimensions, where a bunch of $n$ spins live on each lattice-site and interact among each other as well as with the spins living on neighbouring sites. The reason for writing down the most general Hamiltonian is that, most of our analysis is independent of details like dimension, lattice and spin.

Let $\mathbf{S}_{\mu, \mathbf{x}}(\mu=1,2, \cdots, n)$ be the spins living at the site $\mathbf{x}$. Define,

$$
\mathbf{T}_{\mathbf{x}}=\sum_{\mu=1}^{n} \mathbf{S}_{\mu, \mathbf{x}}
$$

Then the Hamiltonian is defined as,

$$
H=\frac{J^{\prime}}{2} \sum_{\mathbf{x}} \mathbf{T}_{\mathbf{x}}^{2}+J \sum_{\langle\mathbf{x}, \mathbf{y}\rangle} \mathbf{T}_{\mathbf{x}} \cdot \mathbf{T}_{\mathbf{y}}-B \sum_{\mathbf{x}} T_{\mathbf{x}}^{z},
$$

where $\langle$,$\rangle denote nearest neighbours. We take both the couplings to be positive. The models mentioned previously$ are particular cases of the above Hamiltonian. For the Gelfand ladder and the three-leg ladder in HMT, the underlying lattice is a simple chain and $n=2$ and 3 respectively. Both for the modified Shastry-Sutherland model $\mathbf{l}^{\mathbf{5}}$ and the 3-d model of Sutherland ${ }^{\underline{7}} n=2$. For the former the underlying lattice is square and for the latter it is one-fourth depleted cubic.

The variables $T_{\mathbf{x}}^{a}$ 's are also $S U(2)$ generators, and they can take the representations having the Casimir $\mathbf{T}_{\mathbf{x}}^{2}=j(j+1)$, such that $j=0,1, \cdots, n S$, when $n S$ is an integer; and $j=\frac{1}{2}, \frac{3}{2}, \cdots, n S$, when $n S$ is a half-odd integer. Thus the bunch of spins at each site kinematically act respectively like a bosonic or fermionic rotor, except that the values $j$ can take are truncated at $n S$. The Hamiltonian in Eq. (4), feels the value of the underlying spin only through this truncation. This suggests that one can treat the problem for general values of spin.

Now we will use a method of divide-and-conquer to solve for the ground state. If one can write $H$ as a sum over smaller units $h_{i}$, such that there exists a state $\left|\psi_{0}\right\rangle$ which is simultaneously a ground state of each and every $h_{i}$, then $\left|\psi_{0}\right\rangle$ will be a ground state of $H$. To this end, we rewrite $H$ as a sum over all the bonds of the underlying lattice. Let 


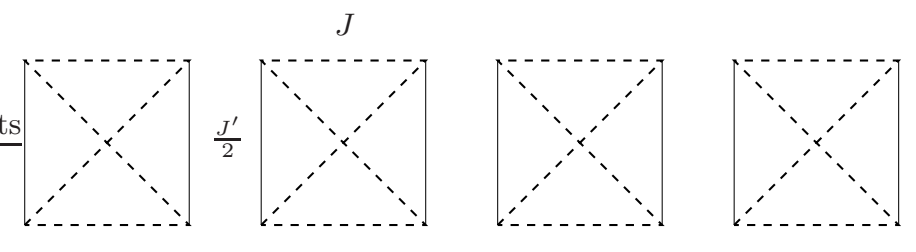

FIG. 2: Writing the ladder Hamiltonian as a sum over units of two dimers.

$i$ label the bonds and let $z$ be the co-ordination number of the lattice. Then,

$$
H=\sum_{i} h_{i}, \quad h_{i}=\frac{J^{\prime}}{2 z}\left(\mathbf{T}_{1, i}^{2}+\mathbf{T}_{2, i}^{2}\right)+J \mathbf{T}_{1, i} \cdot \mathbf{T}_{2, i}-\frac{B}{z}\left(T_{1, i}^{z}+T_{2, i}^{z}\right) .
$$

where $\mathbf{T}_{1, i}$ and $\mathbf{T}_{2, i}$ are the two degrees of freedom living on the bond $i$. Note that the same spin will be shared by many bonds and hence labeled in more ways than one. For the Gelfand ladder, the above break-up is schematically represented in Fig 2

Now we will concentrate on a particular $h_{i}$, and find its ground state and energy as a function of $B$. For notational convenience we shall drop the index $i$ from hereon. After a rearrangement of terms, the bond Hamiltonian can then be rewritten as,

$$
h=\frac{1}{2 z}\left(J^{\prime}-z J\right)\left(\mathbf{T}_{1}^{2}+\mathbf{T}_{2}^{2}\right)+\frac{J}{2}\left(\mathbf{T}_{1}+\mathbf{T}_{2}\right)^{2}-\frac{B}{z}\left(T_{1}^{z}+T_{2}^{z}\right) .
$$

The four terms in $h$ mutually commute and therefore the spectrum is trivially solved. Let $j_{1}\left(j_{1}+1\right), j_{2}\left(j_{2}+1\right)$, $j(j+1)$ and $m$ be the eigenvalues of $\mathbf{T}_{1}^{2}, \mathbf{T}_{2}^{2},\left(\mathbf{T}_{1}+\mathbf{T}_{2}\right)^{2}$ and $\left(T_{1}^{z}+T_{2}^{z}\right)$ respectively. Here $\left|\left(j_{1}-j_{2}\right)\right| \leq j \leq\left(j_{1}+j_{2}\right)$ and $-j \leq m \leq j$. Then the spectrum for $h$ is given by,

$$
E\left(j_{1}, j_{2}, j, m\right)=\frac{1}{2 z}\left(J^{\prime}-z J\right)\left(j_{1}\left(j_{1}+1\right)+j_{2}\left(j_{2}+1\right)\right)+\frac{J}{2} j(j+1)-\frac{B}{z} m .
$$

Since the magnetic field couples to a conserved quantity, the problem of finding the ground state at a given field strength reduces to finding the lowest energy state in each $j$-sector at zero field, i.e., we need to minimize the fieldindependent part of $E$ with respect to $j_{1}$ and $j_{2}$. For non-zero field, the degeneracy arising from the $S U(2)$ symmetry will be lifted and the ground state will have $m=j$. Next we consider the case $J^{\prime}>z J$, for which the coefficient of the first term in $E$ is positive.

When $n S$ is an integer, $j_{1}, j_{2}=0,1, \cdots, n S$ and for half-odd integer values, $j_{1}, j_{2}=\frac{1}{2}, \frac{3}{2}, \cdots, n S$. In both the cases, $j=0,1, \cdots, 2 n S$. Anticipating the solutions, we divide all possible cases into two:

1. Even $j$, integer $n S$;

Odd $j$, half-odd integer $n S$.

2. Odd $j$, integer $n S$;

Even $j$, half-odd integer $n S$.

For Case 1, $E$ is minimized by the choice,

$$
j_{1}=j_{2}=\frac{j}{2}
$$

And for Case 2, the minimum of $E$ is when,

$$
j_{1}=\frac{(j-1)}{2}, \quad j_{2}=\frac{(j+1)}{2} .
$$

There is one exception to the above general rule, though. When $n S$ is half-odd integer and $j=0$, Eqs. (9) give $j_{1}=-\frac{1}{2}$ and $j_{2}=\frac{1}{2}$. This is not an admissible solution and the correct solution is, $j_{1}=j_{2}=\frac{1}{2}$. We will have more to say about this exceptional case later. by,

The ground state energies in the presence of field corresponding to the solutions (8) and (9) are respectively given

$$
\begin{aligned}
& E_{0}^{(1)}=\frac{1}{4 z}\left(J^{\prime}-z J\right) j(j+2)+\frac{J}{2} j(j+1)-\frac{B}{z} j, \\
& E_{0}^{(2)}=\frac{1}{4 z}\left(J^{\prime}-z J\right)(j+1)^{2}+\frac{J}{2} j(j+1)-\frac{B}{z} j .
\end{aligned}
$$


Let $|l, m\rangle_{\alpha}$ denote the state with eigenvalues $l(l+1)$ and $m$ for the operators $\mathbf{T}_{\alpha}^{2}$ and $T_{\alpha}^{z}$ respectively. Then the corresponding ground states are,

$$
\begin{aligned}
& \left|\psi_{0}^{(1)}(j)\right\rangle=|j / 2, j / 2\rangle_{1} \otimes|j / 2, j / 2\rangle_{2}, \\
& \left|\psi_{0}^{(2)}(j)\right\rangle=|(j-1) / 2,(j-1) / 2\rangle_{1} \otimes|(j+1) / 2,(j+1) / 2\rangle_{2} .
\end{aligned}
$$

Since the field-independent part of $E_{0}^{(1,2)}$ are monotonically increasing functions of $j$, as $B$ is increased, $j$ will go up sequentially in steps. The value of the field at which the total spin goes up to $(j+1)$ from $j$ can be obtained from Eqs. (10) and (11). For Case 1, the transition field is,

$$
B_{c_{j}}^{(1)}=\frac{\left(J^{\prime}+z J\right)}{2} j+J^{\prime}
$$

For Case 2, it is given by,

$$
B_{c_{j}}^{(2)}=\frac{\left(J^{\prime}+z J\right)}{2}(j+1) .
$$

This completes the solution of the bond-Hamiltonian $(h)$ for $J^{\prime}>z J$. The important point to note is that the ground states (12) and (13) are product states of the two sites connected by the bond. In the context of the full lattice, this means that we can put together these states living on the bonds and construct a state which will be a simultaneous ground state of $h_{i}$ for every $i$. Consequently, it will be a ground state of the full Hamiltonian, $H$.

For Case 1, the ground state consists of all dimers having total spin $j / 2$ and maximal polarization in the $z$-direction, i.e., $\mathbf{T}_{\alpha}^{2}=(j / 2)(j / 2+1)$ and $T_{\alpha}^{z}=j / 2$. (Note that $j$ denotes the total spin on the bonds, which are not good quantum numbers. Here we merely use $j$ to label the ground states). Since the ground state is unique for individual bond Hamiltonians, this state has to be the unique ground state of the full Hamiltonian. The state can be written as,

$$
\left|\Psi_{0}^{(1)}(j)\right\rangle=\prod_{\mathbf{x}}|j / 2, j / 2\rangle_{\mathbf{x}}
$$

For Case 2, the ground state can be written as,

$$
\left|\Psi_{0}^{2}(j)\right\rangle=\prod_{\mathbf{x} \in A}|(j-1) / 2,(j-1) / 2\rangle_{\mathbf{x}} \prod_{\mathbf{y} \in B}|(j+1) / 2,(j+1) / 2\rangle_{\mathbf{y}}
$$

where $A$ and $B$ denote the two sub-lattices. The above ground state breaks the translation symmetry and is therefore doubly degenerate, the other ground state being the one in which the two sub-lattices are interchanged. Eqs. (16) and (17) give the ground states for all values of $j$ ranging from 0 to $2 n S$. The fractions of the total magnetization at which the plateaus form are,

$$
M=\frac{j}{2 n S},
$$

and the transition fields are given by Eqs. (14) and 15). At each jump of the magnetization, dimers belonging to one of the sub-lattices alternately get excited to a total spin one unit higher. These plateau transitions are similar to

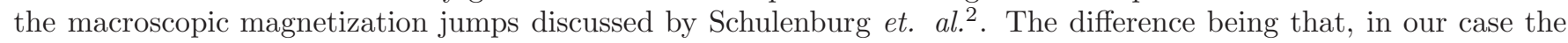
localization of magnons arises from conservation laws.

The above solution for the ground state hinges on one crucial aspect - the ground state of the bond Hamiltonian $(h)$ is a product state on the two sites. For half-odd integer $n S$ and $j=0$, we have seen that the ground state of $h$ has $j_{1}=j_{2}=\frac{1}{2}$. This is not a product state and thus cannot necessarily be satisfied for all bonds. When $n S$ is half-odd integer, the minimum spin on any site is $\frac{1}{2}$ and therefore at zero field the system will be in the ground state of spin- $\frac{1}{2}$ system on the underlying lattice. This is gapless, even in one-dimension ${ }^{10}$, and therefore the magnetization will vary continuously with the field. For $j=1$, the solution again has $j_{1}=j_{2}=\frac{1}{2}$. This is the fully polarized state of the spin- $\frac{1}{2}$ system. The field at which it becomes fully polarized can be determined by the gap of one-magnon states (i.e., all spins except one pointing up). For a hyper-cubic lattice in $D$ dimensions, this is calculated to be,

$$
\frac{B_{c}}{J}=4 S D
$$

Our general expressions for the magnetization fractions (Eq. (18) and transition fields (Eqs. (14), (15) and (19)) check correctly with the results in HMT for spin- $\frac{1}{2}$, two and three-leg ladders. 
A comment is in order here regarding the ground states at plateau transitions. Suppose the field is tuned to $B_{c_{j}}$, i.e. the transition field at which $j$ goes up to $j+1$. Also let $j$ be even (a similar argument holds for odd $j$ as well). Then the state of each bond can be any one of the following three states.

$$
\begin{aligned}
\left|\tilde{\psi}_{0}(j)\right\rangle_{1} & =|j / 2, j / 2\rangle \otimes|j / 2, j / 2\rangle, \\
\left|\tilde{\psi}_{0}(j)\right\rangle_{2} & =|j / 2, j / 2\rangle \otimes|(j / 2+1),(j / 2+1)\rangle, \\
\left|\tilde{\psi}_{0}(j)\right\rangle_{3} & =|(j / 2+1), \quad(j / 2+1)\rangle \otimes|j / 2, j / 2\rangle .
\end{aligned}
$$

This gives rise to a degree of degeneracy which grows exponentially with the system size. In HMT, it is mentioned that the ground state of the spin- $\frac{1}{2}$ ladder is either all triplets (not necessarily polarized) or a mix of singlets and fully polarized triplets. They make an exception for those special values of the field at which there is a jump in magnetization. The above analysis shows that even at transition fields the ground states belong to the afore-mentioned set.

\section{BEYOND THE EXACT REGIME}

The divide-and-conquer method we employed in the previous section to find the exact ground state worked because the ground state of the bond Hamiltonian $(h)$ is a product state. This is no longer true for $J^{\prime}<z J$. Then the ground state of $h$ for a particular $j$ will have,

$$
\mathbf{T}_{1}^{2}=n S(n S+1), \quad \mathbf{T}_{2}^{2}=n S(n S+1), \quad\left(\mathbf{T}_{1}+\mathbf{T}_{2}\right)^{2}=j(j+1), \quad\left(T_{1}^{z}+T_{2}^{z}\right)=j,
$$

This state is not a product state unless $j=n S$. But this does not necessarily mean that the product states cease to be the ground state of $H$ for all $J^{\prime}<z J$. Now we will argue that the product states, and hence the magnetization plateaus, do indeed survive for a while as $J^{\prime}$ goes below $z J$. Significantly, the solutions are not same as that for $J^{\prime}>z J$ as given by Eqs. (16) and (17). The new solutions also form plateaus at the same fractions and it results in a first order phase transition on each plateau (with certain exceptions) at $J^{\prime}=z J$.

For the proof of the existence of product state solutions for $J^{\prime}<z J$, we do the following. First we will show that, at $J^{\prime}=z J$, the only solutions are a finite number of product states, except at the transition fields. Then, by assuming these states to be gapped, it will follow that there exists a positive non-zero $\Delta$ such that the ground state at $(z-\Delta) J<J^{\prime}<z J$ will be among the ground states at $J^{\prime}=z J$.

At $J^{\prime}=z J$, the bond Hamiltonian in Eq. (6) becomes,

$$
h=\frac{J}{2}\left(\mathbf{T}_{1}+\mathbf{T}_{2}\right)^{2}-\frac{B}{z}\left(T_{1}^{z}+T_{2}^{z}\right) .
$$

The above Hamiltonian sees only the total spin of the bond, but is independent of the spins of the sites. Then the ground states of a given $j$-sector are highly degenerate - there is a freedom to choose $j_{1}$ and $j_{2}$ as long as they combine to form total spin $j$. Among these states, there is a subset given by,

$$
\left|\psi_{0}\right\rangle_{p}=|l, l\rangle_{1} \otimes|(j-l),(j-l)\rangle_{2}, \quad l=0,1, \cdots, j,
$$

which constitutes all the only product states. Next we will show that the ground states of the full system at $J^{\prime}=z J$ can be written as product states on the sites. Let us consider two neighbouring bonds - $a$ connecting sites 1 and 2 , and $b$ connecting sites 2 and 3 . Also let $h_{a}$ and $h_{b}$ be the corresponding bond Hamiltonians. Then, at $J^{\prime}=z J$ any ground state, of $\left(h_{a}+h_{b}\right)$ has to satisfy the following conditions.

$$
\begin{aligned}
\left(\mathbf{T}_{1}+\mathbf{T}_{2}\right)^{2}\left|\phi_{0}\right\rangle & =j(j+1)\left|\phi_{0}\right\rangle, \\
\left(\mathbf{T}_{1}^{z}+\mathbf{T}_{2}^{z}\right)\left|\phi_{0}\right\rangle & =j\left|\phi_{0}\right\rangle, \\
\left(\mathbf{T}_{2}+\mathbf{T}_{3}\right)^{2}\left|\phi_{0}\right\rangle & =j(j+1)\left|\phi_{0}\right\rangle, \\
\left(\mathbf{T}_{2}^{z}+\mathbf{T}_{3}^{z}\right)\left|\phi_{0}\right\rangle & =j\left|\phi_{0}\right\rangle,
\end{aligned}
$$

where $j$ is determined by the field, $B$. In the sub-space of fixed eigenvalues for $\mathbf{T}_{1}^{2}, \mathbf{T}_{2}^{2}$ and $\mathbf{T}_{3}^{2}$, Eqs. (26) and (27) imply that,

$$
\left|\phi_{0}\right\rangle=\left|\phi_{0}\right\rangle_{12} \otimes\left|\phi_{0}\right\rangle_{3}
$$


where, $\left|\phi_{0}\right\rangle_{12}$ lives in the combined Hilbert space of the sites 1 and 2 , and $\left|\phi_{0}\right\rangle_{3}$ lives in the Hilbert space of the site 3. This follows from the fact that for fixed values of $j_{1}$ and $j_{2}$, there is a unique state in the Hilbert space of sites 1 and 2 which satisfies Eqs. (26) and (27). Similarly Eqs. (28) and (29) imply that,

$$
\left|\phi_{0}\right\rangle=\left|\phi_{0}\right\rangle_{1} \otimes\left|\phi_{0}\right\rangle_{23}
$$

where, $\left|\phi_{0}\right\rangle_{1}$ lives in the Hilbert space of the site 1 and $\left|\phi_{0}\right\rangle_{23}$ lives in the combined Hilbert space of the sites 2 and 3. Finally, Eqs. (30) and (31) imply that,

$$
\left|\phi_{0}\right\rangle=\left|\phi_{0}\right\rangle_{1} \otimes\left|\phi_{0}\right\rangle_{2} \otimes\left|\phi_{0}\right\rangle_{3}
$$

i.e., $\left|\phi_{0}\right\rangle$ is a product state of the three sites. Since every site is part of at least two bonds, this necessarily implies that the ground states of $H$ at $J^{\prime}=z J$ are product states. These ground states can be written as,

$$
\left|\Psi_{0}\left(J^{\prime}=z J, j\right)\right\rangle=\prod_{\mathbf{x} \in A}|l, l\rangle_{\mathbf{x}} \otimes \prod_{\mathbf{y} \in B}|j-l, j-l\rangle_{\mathbf{y}}, \quad l=0,1, \cdots, j,
$$

What we have shown now is that, though the bond Hamiltonian $(h)$ admits solutions which are not product states at $J^{\prime}=z J$, such solutions are not admissible for the full Hamiltonian $(H)$.

At transition fields, there is a greater degree of degeneracy. Then the constraint on the ground state is more relaxed - the total spin on a bond can be $j$ or $j+1$. This gives rise to a degeneracy which is local and hence grows exponentially with the system size. Away from the transitions fields, the ground states are those given by Eq. (33) and are not related to each other by any local transformation. It is then fair to assume that there is a gap to excitations.

Now let us see why gap at $J^{\prime}=J$ implies the existence of product ground state for $(z-\Delta) J<J^{\prime}<2 J$, for some positive $\Delta$. The three terms in $H$ in Eq. (4) mutually commute. This means that one can choose the eigenstates to be independent of the three parameters $J^{\prime}, J$ and $B$. The energy spectrum will depend linearly on all the three parameters and all transitions of the ground state are via level crossings. (The transitions will be first order unless there is a continuum of level crossings - a possibility in the thermodynamic limit). A gap to the ground states at $J^{\prime}=z J$ would then imply that there exists some non-zero positive number $\Delta$, such that for $(z-\Delta) J<J^{\prime}<z J$ the ground state(s) will be among those at $J^{\prime}=z J$. Among the states in Eq. (33), the one with the lowest energy for $J^{\prime}<z J$ is given by,

$$
\begin{aligned}
& \left|\Psi_{0}^{<}(j)\right\rangle=\prod_{i \in A}|j, j\rangle \otimes \prod_{k \in B}|0,0\rangle, \quad \text { for } j \leq 2 S, \\
& \left|\Psi_{0}^{<}(j)\right\rangle=\prod_{i \in A}|2 S, 2 S\rangle \otimes \prod_{k \in B}|(j-2 S),(j-2 S)\rangle, \quad \text { for } j>2 S .
\end{aligned}
$$

These states also have the same fractions for magnetization as before. But, now the system prefers to put all the spin into sites in one sub-lattice, if that is possible. This is in contrast to the situation for $J^{\prime}>z J$ where it is energetically favourable to distribute the spin equally among the two sub-lattices. At $j=2 S$, one sub-lattice will be saturated and then the other sub-lattice start getting excited to higher and higher spins.

In the above states, when $j>2 S$, sites in one sub-lattice have spin $2 S$ and those in the other sub-lattice have spin $(j-2 S)$. On the plateau, each spin is maximally polarized in the $z$-direction. Below a critical field, gapless phase of the same set of spins will have lower energy. The critical field, in units of $J$, will be the gap in the spectrum of one-magnons (states with all spins but one maximally polarized, the odd one having $z$-component one less than maximum). For a hyper-cubic lattice in $D$-dimensions consisting of spins $j_{1}$ in one sub-lattice and $j_{2}$ in the other, the critical field is given by,

$$
\frac{B_{c r i t}}{J}=2 D\left(j_{1}+j_{2}\right)
$$

HMT have numerically found such lines of transition for $S=\frac{1}{2}$, two and three legged ladders and the formula above is consistent with their results. For the two-leg ladder, the transition occurs when the fully polarized effective spin-1 chain becomes gapless. Then $j_{1}=j_{2}=1$, and $B_{\text {crit }}=4 J$. For the three-leg ladder there are three cases $\left.-a\right) j_{1}=\frac{1}{2}$, $\left.j_{2}=\frac{1}{2}, b\right) j_{1}=\frac{1}{2}, j_{2}=\frac{3}{2}$ and $\left.c\right) j_{1}=\frac{3}{2}, j_{2}=\frac{3}{2}$. Then the transitions occur respectively at $B_{c r i t}=2 J, 4 J$ and $6 J$.

To summarize our results thus far, we first solved for the ground states of the general spin- $S$ model in arbitrary dimensions for $J^{\prime}>z J$. The product state solutions we obtain exhibit magnetization plateaus. Then we argued that there exist product state solutions and hence magnetization plateaus even as $J^{\prime}$ goes below $z J$, but the mechanism for plateau formation is different from the case $J^{\prime}>z J$. Thus, on a plateau, there are first order phase transitions at $J^{\prime}=z J$. 


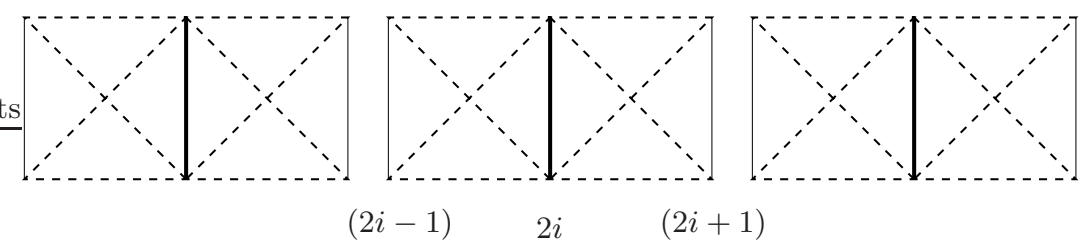

FIG. 3: Writing the Hamiltonian as a sum over units of three dimers. Bold full lines represent bonds of strength $J^{\prime}$, thin full lines $J^{\prime} / 2$ and dashed lines $J$.

HMT have obtained a similar first order phase transition on the plateau for the three-leg, $S=\frac{1}{2}$ model. In their case the transition occurs on $M=\frac{1}{3}$ plateau from a fully polarized $S=\frac{1}{2}$ chain to an alternating chain consisting of spins $\frac{1}{2}$ and $\frac{3}{2}$. The latter is not a simple product state.

The plateau solutions for $J^{\prime}>2 J$, given by Eqs. (16) and (17), and that for $J^{\prime}<2 J$, given by Eqs. (34) and (35), coincide for $j=0,1,(2 n S-1)$ and $2 n S$. For $S=\frac{1}{2}$ ladder $(n=2)$, all the plateaus belong to the above set and therefore there is no first order transition at $J^{\prime}=2 J$ along any of the plateaus.

From now we will concentrate on the two-leg ladder. First we will show for some higher spin cases that first order transitions along the plateaus at $J^{\prime}=2 J$ does indeed exist.

\section{PHASE TRANSITIONS FOR THE LADDER AT $J^{\prime}=2 J$}

We have seen that the ground states of the bond Hamiltonian are not product states for $J^{\prime}<2 J$. Therefore, to look for product state solutions in this range, we try a different divide-and-conquer scheme involving bigger units. To write $H_{l}$ as sum over units containing three dimers then suggests itself ( see Fig. [3),

$$
\begin{aligned}
& H_{l}=\sum_{i} \tilde{h}_{i}, \\
& \tilde{h}_{i}=\frac{J^{\prime}}{4}\left(\mathbf{T}_{2 i-1}^{2}+2 \mathbf{T}_{2 i}^{2}+\mathbf{T}_{2 i+1}^{2}\right)+J \mathbf{T}_{2 i} \cdot\left(\mathbf{T}_{2 i-1}+\mathbf{T}_{2 i+1}\right)-\frac{B}{2}\left(T_{2 i-1}^{z}+2 T_{2 i}^{z}+T_{2 i+1}^{z}\right) .
\end{aligned}
$$

As before, we drop the index $i$ and write the 3-dimer Hamiltonian as,

$$
\tilde{h}=\frac{J^{\prime}}{4}\left(\mathbf{T}_{1}^{2}+2 \mathbf{T}_{2}^{2}+\mathbf{T}_{3}^{2}\right)+J \mathbf{T}_{2} \cdot\left(\mathbf{T}_{1}+\mathbf{T}_{3}\right)-\frac{B}{2}\left(T_{1}^{z}+2 T_{2}^{z}+T_{3}^{z}\right) .
$$

$\mathbf{T}_{1}^{2}, \mathbf{T}_{2}^{2}, \mathbf{T}_{3}^{2},\left(\mathbf{T}_{1}+\mathbf{T}_{3}\right)^{2}$ and $\left(\mathbf{T}_{1}^{z}+\mathbf{T}_{2}^{z}+\mathbf{T}_{3}^{z}\right)$ are mutually commuting conserved quantities and this essentially reduces the problem to that of two (variable) interacting spins coupled to a magnetic field of unequal strengths at the two sites. Unfortunately, the spectrum cannot be found analytically for arbitrary values of spin.

We have numerically diagonalized $\tilde{h}$ for spins up to 3 . We have found that the product state solutions given in Eqs. (34) and (35) do indeed exist for some $J^{\prime}<2 J$. (This diagonalization can be easily performed for higher spins also, but we chose to restrict it to reasonable values). Note that the existence of the product state solutions for $\tilde{h}$ necessarily implies their existence for the full ladder. This in turn confirms our prediction of first order transitions on a plateau at $J^{\prime}=2 J$.

Next we study the particular case $S=1$. We numerically analyse it to obtain the phase diagram in the complete parameter space and thus confirm our analytic results.

\section{SPIN-1 LADDER}

The spin- $\frac{1}{2}$ ladder was numerically studied in great detail by HMT to obtain the complete phase diagram. Our results for $J^{\prime}>2 J$ and the arguments for the existence of the plateaus beyond the exact regime are consistent with their phase diagram. As we mentioned in the previous section, to obtain the new phase predicted by us, one needs to look at spins higher than $\frac{1}{2}$. In this section we present the results of an exact diagonalization analysis of the spin- 1 ladder.

Since the individual dimer spins are conserved, we need to analyse only effective spin chains consisting of spins of varying values at each site. We have employed the Lanczos algorithm to exactly diagonalize the Hamiltonian and 


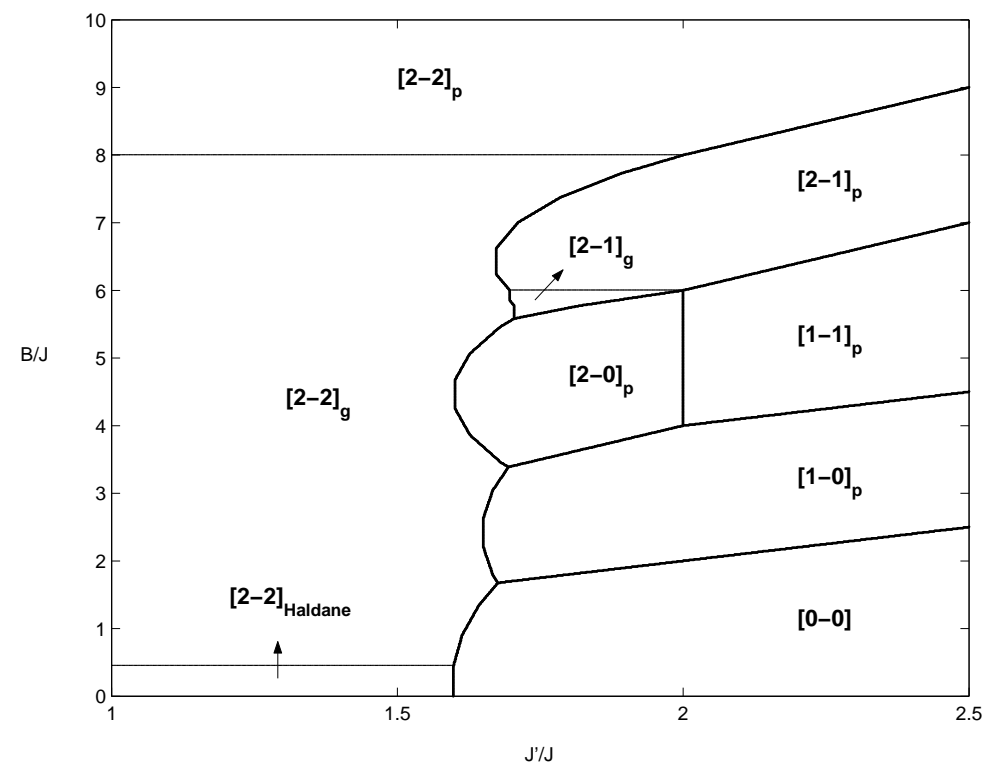

FIG. 4: Phase diagram for the spin-1 ladder. Thick and thin lines denote first and second order transitions respectively.

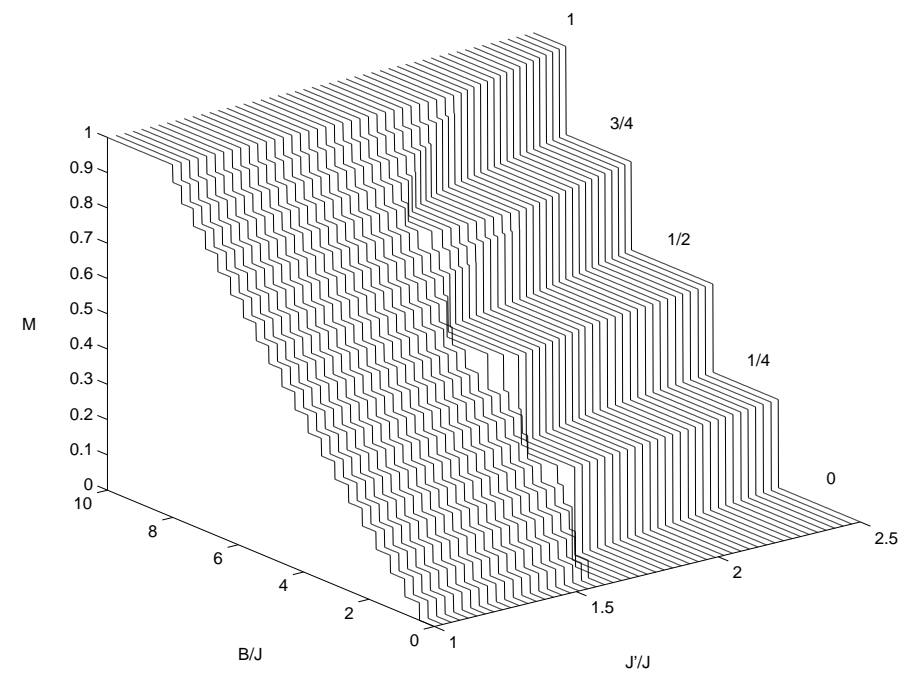

FIG. 5: Magnetization curves for the spin-1 ladder. The fractional values of magnetization are denoted on the plateaus.

obtain the ground state energies. We have worked with 10 dimers (20 spins) and used periodic boundary conditions. For the finite system that we studied, we have checked that all the relevant states are such that the spins of the dimers belonging to a given sub-lattice are the same. There are exceptions to this at plateau transitions, arising from the local degeneracy which has been discussed in Section [II In the phase diagram (see Fig. 4), $\left[j_{1}-j_{2}\right]_{p}$ and $\left[j_{1}-j_{2}\right]_{g}$ respectively denote the fully polarized and the gapless phases of an alternating chain consisting of spins $j_{1}$ and $j_{2}$. In Fig. [5 the magnetization is plotted as a function of $B / J$ for various values of $J^{\prime} / J$.

For $J^{\prime}>2 J$ the analytic results from Section 1 are very accurately reproduced. Since all the relevant states are product states, there are no finite-size corrections and agreement with the analytic results is exact up to numerical inaccuracies. There are five plateaus at the fractions $0, \frac{1}{4}, \frac{1}{2}, \frac{3}{4}$ and 1 . The successive transitions occur at the 
following values of the field,

$$
\begin{aligned}
\frac{B_{c_{1}}}{J}=\frac{J^{\prime}}{J}, & \frac{B_{c_{2}}}{J}=2+\frac{J^{\prime}}{J}, \\
\frac{B_{c_{3}}}{J}=2+2 \frac{J^{\prime}}{J}, & \frac{B_{c_{4}}}{J}=4+2 \frac{J^{\prime}}{J} .
\end{aligned}
$$

On the $M=\frac{1}{2}$ plateau, $[1-1]_{p}$ phase gives way to $[2-0]_{p}$ at $J^{\prime}=2 J$, as we expect from our analysis in Section [II] From $[2-1]_{p}$ and $[2-2]_{p}$ phases there are second order transitions to the corresponding gapless phases $[2-1]_{g}$ and $[2-2]_{g}$ respectively. The lines of these transitions, obtained from Eq. (36), are given by $B_{\text {crit }}=6 \mathrm{~J}$ and $8 \mathrm{~J}$ respectively. These lines meet the lines separating plateaus, given in Eq. (41) at $J^{\prime}=2 J$.

For $J^{\prime} \ll 2 J$, all the dimers have spin 2 . Then the ground state at zero field is the Haldane phase of the uniform spin-2 chain 11.12 . When the field is turned on, the magnetization remains zero till a critical field at which the system becomes gapless 13 . Then with further increase in field, the magnetization continuously increases and finally reaches saturation $\left([2-2]_{p}\right)$. In the plot for magnetization curves in Fig 5 there are discernible steps even in the gapless phases $[2-1]_{g}$ and $[2-2]_{g}$. These are spurious jumps arising from finite-size effects. To obtain more accurate results, it will be necessary to employ techniques like DMRG.

In the intermediate range, i.e. around $J^{\prime} \approx 1.6 J$, just like in the $S=\frac{1}{2}$ case in HMT, with changing field, the system goes in and comes out of various gapped and gapless phases. At zero field, $[0-0]$ phase goes into spin-2 Haldane phase at $J^{\prime} \approx 1.5975 J$.

For higher spins, there will be $(4 S+1)$ plateaus for $J^{\prime}>2 J$ as discussed in Section III First order transitions of the type, $[1-1]_{p}$ to $[2-2]_{p}$ will then happen at all plateaus except the first two and the last two. There will also be second order transitions of the type $[2-1]_{p}$ to $[2-1]_{g}$, between $[2 S-(j-2 S)]_{p}$ and $[2 S-(j-2 S)]_{g}$ for $(2 S+1) \leq j \leq 4 S$.

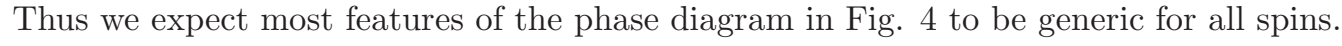

\section{SUMMARY AND CONCLUSION}

We have generalized a class of existing spin- $\frac{1}{2}$ models in various dimensions, which exhibit magnetization plateaus, to arbitrary values of spin. By using simple techniques we are able to analytically solve the spin- $S$ model in a substantial regime of the parameter space. Our approach also throws light upon the phases beyond the exact regime. In particular, we are able to predict that there will be a new type of plateau phase. Such phases cannot exist for the spin- $\frac{1}{2}$ ladders studied in HMT. We have tested our results numerically for the particular case of $S=1$ two-leg ladder.

Though our general model is defined on arbitrary bipartite lattices, each site has a sub-structure which includes $n$ spins. Therefore, constructing physically feasible models, where bonds of equal strength have equal length, is a non-trivial task. As far as we know, the one-dimensional linked tetrahedra introduced by Gelfand ${ }^{3}$ and Sutherland's generalization of the same to $3-d$ are the only possibilities.

\section{ACKNOWLEDGMENTS}

We thank Diptiman Sen and R. Shankar for very useful discussions. V.R.C. thanks Council for Scientific and Industrial Research, India for a Junior Research Fellowship. N.S. acknowledges Department of Science and Technology, India for financial support through Grant No. SP/S2/M-11/2000.

1 D.C. Cabra, M.D. Grynberg, A. Honecker, P. Pujol, Condensed Matter Theories (Vol 16), Ed. S. Hernandez and J.W. Clark (New York: Nova Science), 17 (2001); Preprint cond-mat/0010376, and references therein.

2 J. Schulenburg, A. Honecker, J. Schnack, J. Richter, H.-J. Schmidt, Phys. Rev. Lett. 88, 167207 (2002).

3 M.P. Gelfand, Phys. Rev. B 43, 8644 (1991).

4 I. Bose, S. Gayen, Phys. Rev. B 48, 10653 (1993).

${ }^{5}$ E. Müller-Hartmann, R.R.P. Singh, C. Knetter, G.S. Uhrig, Phys. Rev. Lett. 84, 1808 (2000).

6 A. Honecker, F. Mila, M. Troyer, Eur. Phys. J. B 15, 227 (2000).

7 B. Sutherland, Phys. Rev. B 62, 11499 (2000).

${ }^{8}$ F. Mila, Eur. Phys. J. B 6, 201 (1998)

9 B.S. Shastry, B. Sutherland, Physica B 108B, 1069 (1981). 
10 H.A. Bethe, Z. Phys. 71, 205 (1931).

11 F.D.M. Haldane, Phys. Lett. 93A, 464 (1983); Phys. Rev. Lett. 50, 1153 (1983).

12 J.B. Parkinson, J.C. Bonner, Phys. Rev. B 32, 4703 (1985).

13 Jizhong Lou, Shaojin Qin, Zhaobin Su, Phys. Rev. B 62, 13832 (2000). 